\title{
Face Recognition using Haar Cascade Classifier
}

\author{
Arnav Madan
}

Department of IT \& Maharaja Agrasen Institute Of Technology,Delhi,India

To Cite this Article

Arnav Madan, "Face Recognition using Haar Cascade Classifier", International Journal for Modern Trends in Science and Technology, Vol. 07, Issue 01, January 2021, pp.- 85-87.

\section{Article Info}

Received on 22-November-2020, Revised on 18-December-2020, Accepted on 28-December-2020, Published on 05-January-2021.

\section{ABSTRACT}

With development of machine learning technology many applications have been revolutionized which earlier usedto utilize high amoun to fresources. Face recognition is a crucial security application. Though this paper we present this application using optimized amount of resources and high efficiency.

Keywords-Face detection, Machine Learning, OpenCV, Haar Cascade Classifier

\section{INTRODUCTION}

The face is crucial for human identity. It is the feature which best distinguishes person. Face recognition is an interesting and challenging problem, and impacts important applications in many areas such as identification for law enforcement, authentication for banking and security system access, and personal identification among others. Face recognition is an easy task for humans but its entirely different task for a computer. A very little is known about human recognition to date on How do we analyze an image and how does the brain encode it and Are inner features (eyes, nose, mouth) router features(head shape, hairline) used for a successful face recognition?Neurophysiologist David Hubel and Torsten Wiesel has shown that our brain has specialized nerve cells responding to specific local features of a scene, such as lines, edges, angles or movement. Since we don "t see the

world as scattered pieces, our visual cortex must some how

combine the different sources of information into useful

patterns.
Automatic face recognition is all about extracting those meaningful features from an image,putting them into a use- ful representation and performing some classifications on them.Face recognition based on the geometric features of a face is probably the most intuitive approach to Human identification. The whole process can be divided in three major steps where the first step is to find a good database of faces with multiple images for each individual.The next step is to detect faces in the database images and use them to train the face recognizer and the last step is to test the face recognizer to recognise faces it was trained for.

\section{STRUCTURE OF PAPER}

The paper is organized as follows: In Section 1, the introduction of the paper is provided along with the structure, important terms, objectives and overall description. In Section 2 we discuss related work. In Section 3 we have the complete information about the development of security system using haar cascade and in section 4 we have Methodology and the process description.

Section 5 tells us about the future scope and concludes the paper with acknowledgement and references. 


\section{OBJECTIVE}

Using Haar Cascade Classifier to recognise and detect face on webcamera. The primary objective of this work is to develop a framework for face detection using Haar Feature-based Cascade Classifiers.Based on the framework developed, it will be extended for the usage in security purposes.

\section{II.IMPLEMENTATION}

\section{A.Importherequiredmodules}

The Modules required to perform the facial recognition are cv2, os, image module and numpy. cv2 is the OpenCV module and contains the functions for face detection and recognition. OS will be used to maneuver with image and directory names. First, we use this module to extract the image names inthe database directory and then from these names individual number is extracted, which is used as a label for the face in that image. Since, the dataset images are in gif format and as of now, OpenCV does not support gif format, Image module from PIL is used to read the image in grayscale format. Numpy arrays are used to store the images

\section{B. Load the face detectionCascade}

To Load the face detection Cascade the first step is to detect the face in each image. Once we get the region of interest containing the face in the image, we use it For training the recognizer. For the purpose of face detection, we will use the Haar Cascade provided by OpenCV. The haar cascades that come with OpenCV are located in the directory of OpenCV installation. haarcascade frontalface default.xml is used for detecting the face. Cascade is loaded using the cv2 CascadeClassifier function which takes the path to the cascade $x m l$ file. if the $\mathrm{xml}$ file is in the current working directory, then relative path is used.

\section{Create the Face RecognizerObject}

The next step involve creating the face recognizer object. The face recognizer object has functions like FaceRecog- nizer.train to train the recognizer and FaceRecognizer.predict to recognize a face. OpenCV currently provides Eigenface Recognizer,Fisherface Recognizer and Local Binary Patterns Histograms Face Recognizer.we have used Local Binary Pat- terns Histograms Face Recognizer to perform face recognition. With Local Binary Patterns it is possible to describe the texture and shape of a digital image. This is done by dividing an image into several small regions from which the features are extracted that can be used to get a measure for the similarity between the images.

D. Prepare the training set Perform thetraining To create the function to prepare the training set, we will define a function that takes the absolute path to the image database as input argument and returns tuple of 2 list, one containing the detected faces and the other containing the corresponding label for that face. For example, if the ith index in the list of faces represents the $4^{\text {th }}$ individual in the database, then the corresponding ith location in the list of labels has value equal to 4 . Now to perform the training using the Face Recognizer.

Train function. It requires 2 arguments, the features which in this case are the images of faces and the corresponding labels assigned to these faces which in this case are the individual number that we extracted from the image names.

\section{E.Testing}

For testing the Face Recognizer, we check if the recognition was correct by comparing the predicted label predicted with the actual label actual. The label actual is extracted using the os module and the string operations from the name of the image. We also display the confidence score for each recognition.

\section{DEVLOPMENT OF SECURITY SYSTEM USING FACE RECOGNITION}

This security system is build to implement on a door. Where every person entered is checked and their images are taken and compared with available data. If the person is found in database, will be authorized to access otherwise access will be denied. Every image taken and their authorization whether authorized or not, will send to Google Drive with time and date of entry. From Google drive data can be getting to use to display on apps or on websites.

To build this security system we will require a Raspberry pi board, Camera,Sensors pair,Infrared Sensors pair,6x2 LCD screen,USB Cables, Ethernet jumper wires and Open CV module. The Raspberry $\mathrm{Pi}$ is a series of credit card-sized single-board computers. It could work on Linux. It has 40 GPIO pins, Ethernet port, 4 USB ports, micro SD card port etc.Camera takes the image and saves it in "Newface ${ }^{\text {ee }}$ folder, from where it will be taken for comparison.A set of IR (infrared) sensors has been set, which detects the coming 
person.IR sensors pair sends signal to raspberry pi, which activates the camera and takes the image of person standing in front of it .LCD screen is used to display the messages. Ethernet wire is used make raspberry pi internet enable to send data to Google drive USB cable powers the raspberry pi.

\section{Metododlogy}

Haarclassification is a tree-based technique where in the training phase, a statistical boosted rejection cascade is created. Boosted means that one strong classifier is created from weak classifiers (see fig. 3), and a weak classifier is one that correctlygets the classification right in at least above fifty percent of the cases. This buildup to a better classifier from many weak is done by increasing the weight (penalty) on misclassified samples so that in the next iteration of training a hypothesis that gets those falsely classified samples right is selected. Finally the convex combination of all hypotheses is computed.

Haar Cascade is a machine learning-based approach where a lot of positive and negative images are used to train the classifier.

- Positive images - These images contain the images which we want our classifier to identify.

- Negative Images - Images of everything else, which do not contain the object we want to detect.

\section{FUTURE SCOPE AND CONCLUSION}

Security is an imperative part of any industry. This work is most particularly for criminal identification. The algorithms carried out in this paper were Viola-Jones algorithm and Linear binary pattern algorithm. The presented system will get implemented using Open $\mathrm{CV}$ and Raspberry pi. The recognition rate attained by this process is $90 \%-98 \%$. There will be deviation in the result on account of the distance, camera resolution and lightning. Advanced processors can be put to use to reduce the processing time. By affixing more number of recognition servers to attenuate the processing time for collection of images.

\section{ACKNOWLEDGEMENT}

Aside from God Almighty, the researchers would like to express their gratitude to their families, relatives, and dear ones whose involvement made a significant impact on the accomplishment of this task.

\section{REFERENCES}

1.https://medium.com/@dipti.rohan.pawar/improving-perf ormance-of-convolutional-neural-network-2ecfe0207de7E.

2. https://arxiv.org/abs/1409.1556

3. https://machinelearningmastery.com/how-to-use-transfe r-learning-when-developing-convolutional-neural-network -models/

4. https://analyticsindiamag.com/practical-comparison-of-t ransfer-learning-models-in-multi-class-image-classificatio $\mathrm{n} /$

5. https://medium.com/abraia/first-steps-with-transfer-lear ning-for-custom-image-classification-with-keras-b941601f cad5
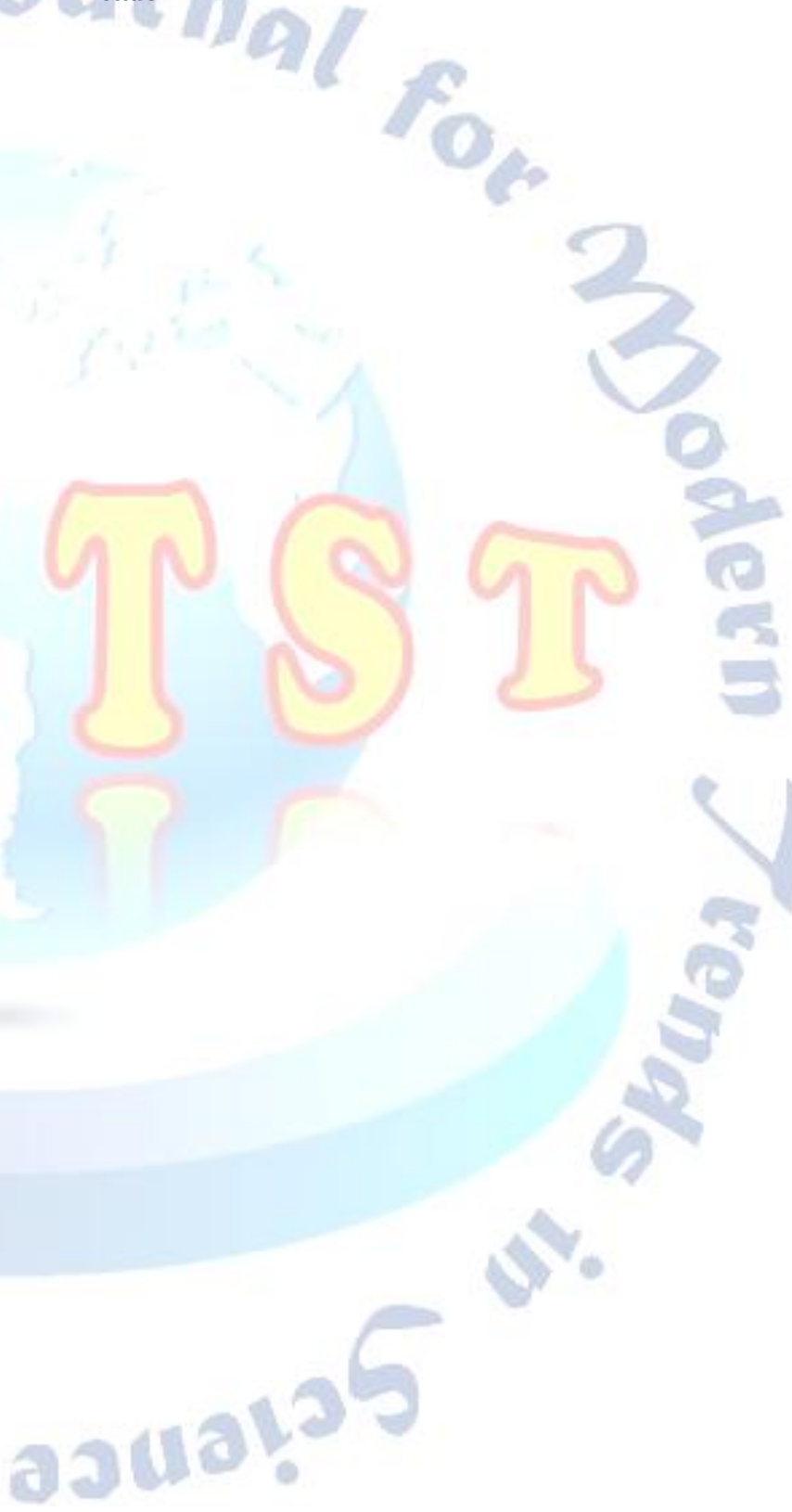